\title{
Effect of Scan Strategy on Mechanical Properties of AlSi12 Lattice Fabricated by Selective Laser Melting
}

\author{
Masahiko Sairaiji $^{1}$, Hiroshi Yoshizaki ${ }^{1}$, Hideaki Iwaoka ${ }^{2}$, Shoichi Hirosawa ${ }^{2}$ and Shoji Maruo ${ }^{2}$ \\ ${ }^{1}$ SOLIZE Products corp., 7-10-1 Chuorinkan, Yamato-shi, Kanagawa 242-0007 Japan \\ E-mail: Masahiko.sairaiji@solize.com \\ ${ }^{2}$ Faculty of Engineering, Yokohama National University, 79-5 Tokiwadai, Hodogaya-ku, Yokohama \\ 240-8501, Japan
}

In this study, the influence of scan strategies, such as scan order and scan patterns, on compressive load capacity was investigated for the aluminum alloy AlSi12-made lattice structures fabricated by selective laser melting. The scan order of concentric scan patterns affected compressive load capacity. Better mechanical properties were obtained when the scan order was set from the outside. Setting the scan order from the inside caused coarsening of the grains at the center of the strut, thus worsening the mechanical properties due to the reduced area fraction of the finegrained regions. The mechanism of such grain coarsening was explained based on the heat transfer direction. Furthermore, the scan pattern also affected the size and orientation of the grains in the lower zone of the strut as well as its geometrical accuracy. A stripe pattern with a rotation of $67^{\circ}$ from layer to layer decreased the geometrical accuracy but increased the hardness of the strut owing to the smaller size and random orientation of the grains in the lower strut zone.

DOI: $10.2961 /$ jlmn.2020.01.2002

Keywords: selective laser melting, lattice structure, aluminum alloy, compressive load, microstructure

\section{Introduction}

Metallic lattice structures have been actively studied owing to their lightweight and industrial applications (such as automotive and aerospace industries). Lattice components are commonly fabricated by additive manufacturing systems because additive manufacturing is suitable for producing complex three-dimensional components [1]. The properties of the components fabricated by additive manufacturing vary with process parameters, namely, laser power, scanning speed, and scan strategy [2, 3]. Scan strategy is a combination of scan pattern and scan order of each layer, and there are numerous scan strategy parameters such as raster, concentric, rotation, mesh, fractal, etc. [2, 3, 4, 5]. Scan strategy has been mainly evaluated on a larger scale for tensile testing of materials. Finer scale testing has not been conducted, especially for lattice structures. In this study, we evaluated the influence of scan strategy on the mechanical properties of lattice structures. We used aluminum alloy powder to fabricate the lattice structure, as aluminum alloys are widely used in automotive and aerospace industries owing to their unique features (i.e., low density, good mechanical properties, and high wear resistance) [6].

\section{Materials and methods}

\subsection{Materials}

We used an AlSi12 alloy, which is one of the most popular aluminum-silicon alloys for casting. Recently, the AlSi12 alloy has been widely used as a powder material for selective laser melting (SLM). The powder size constituted $\mathrm{D} 10=7.6 \mu \mathrm{m}, \mathrm{D} 50=18.3 \mu \mathrm{m}$, and D90 $=34.2 \mu \mathrm{m}$.

\subsection{Methods}

Three scan strategies were used for fabrication, as illustrated in Fig. 1. The first strategy was a concentric scan pattern with the scan order set from the outside (C-OUT). The second employed strategy was a concentric scan pattern with the scan order set from the inside (C-IN). The third scan strategy included a stripe scan pattern with $67^{\circ}$ rotation from layer to layer (STRIPE). The SLM process
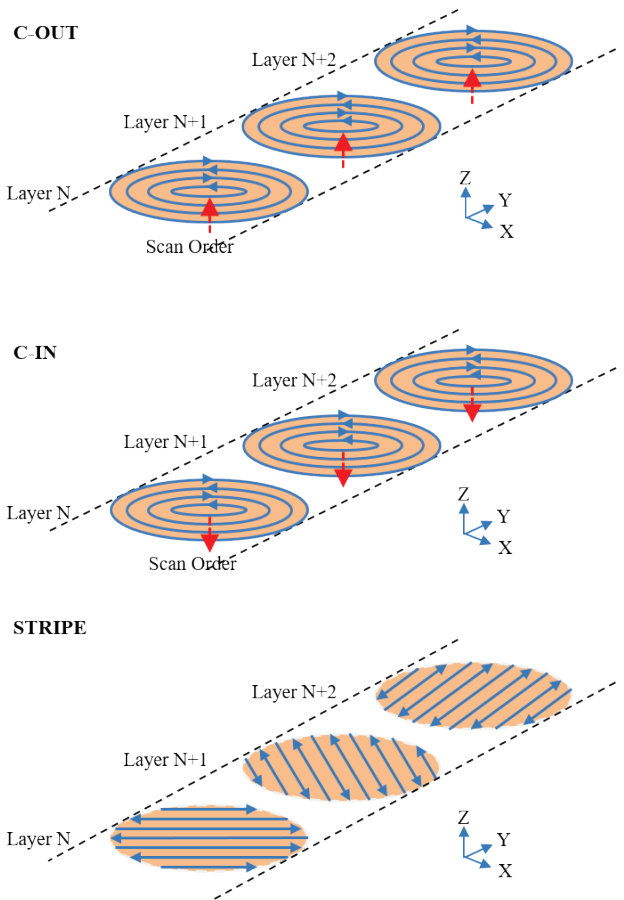

Fig. 1 Scan strategies used in this study 
usually uses two types of scan patterns to fabricate an object. One is a fill scan for the inner zone of the object and the other is a contour scan for the outer skin zone of the object. In this study, only a fill scan was used to clearly distinguish between the effects of scan strategies on a thin strut.

Computer-aided design (CAD) data of the fabricated lattice structure included a body-centered cubic (BCC) structure with a strut diameter of $800 \mathrm{um}$, oriented $35.3^{\circ}$ from the horizontal plane (Fig. 2). (a)

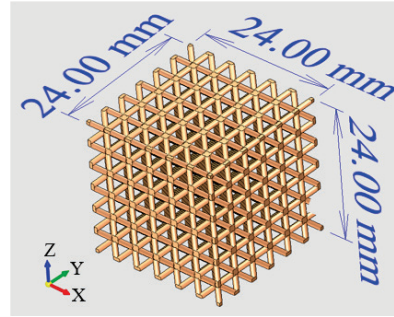

(b)

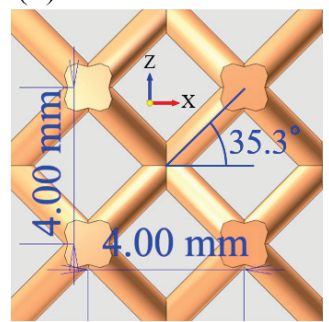

Fig. 2 CAD data of the fabricated lattice structure in: (a) isometric view, (b) enlarged side view

A 3D Systems ProX200 was used as an SLM system for fabrication of the lattice structure. The processing parameters were set at a laser power of $240 \mathrm{~W}$, scanning speed of $1200 \mathrm{~mm} / \mathrm{s}$, hatch spacing between each path of $0.120 \mathrm{~mm}$, and layer thickness of $0.03 \mathrm{~mm}$.

Measurements of the compressive load capacity were conducted both parallel and perpendicular to the built direction using a SHIMADZU Universal testing machine AG-100kNIS. Vickers hardness of the node and strut was measured using a Matsuzawa Vickers hardness tester MMT-X1A with a $0.5 \mathrm{kgf}$ load. KEYENCE optical microscope VHX S550 was used for measuring the geometrical dimensions of the strut cross-section. JEOL scanning electron microscope (SEM) JSM-7001F was used for the investigation of the microstructure and crystal orientation by the electron backscattering diffraction (EBSD) mode. Sample preparation consisted of mechanical polishing by an oxide polishing suspension (OP-S) with colloidal silica serving as an abrasive material.

\section{Results and discussion}

\subsection{Compressive load capacity measurements}

Fig. 3 shows the lattice structure before and after the load testing. The broken point was confirmed at the strut. (a)

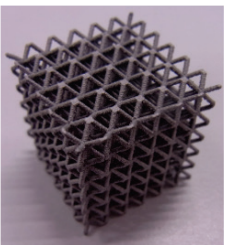

(b)

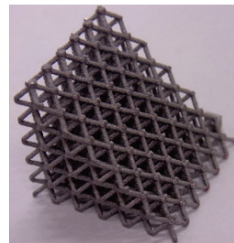

(c)

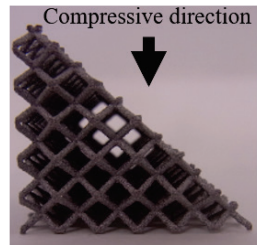

Fig. 3 Overview of a lattice structure fabricated by SLM: (a) before load testing, (b) isometric view after load testing, (c) side view after load testing
Fig. 4 shows the compressive load-displacement curves and the evaluated load capacity in the vertical and horizontal directions. It is clear from Fig. 4 that C-OUT has a higher load capacity than C-IN. However, both concentric strategies have a higher load capacity than STRIPE in both directions of compression. Therefore, first, we clarified the influence of the scan order on the concentric scan pattern, and then, the influence of the scan pattern was revealed.

(a)

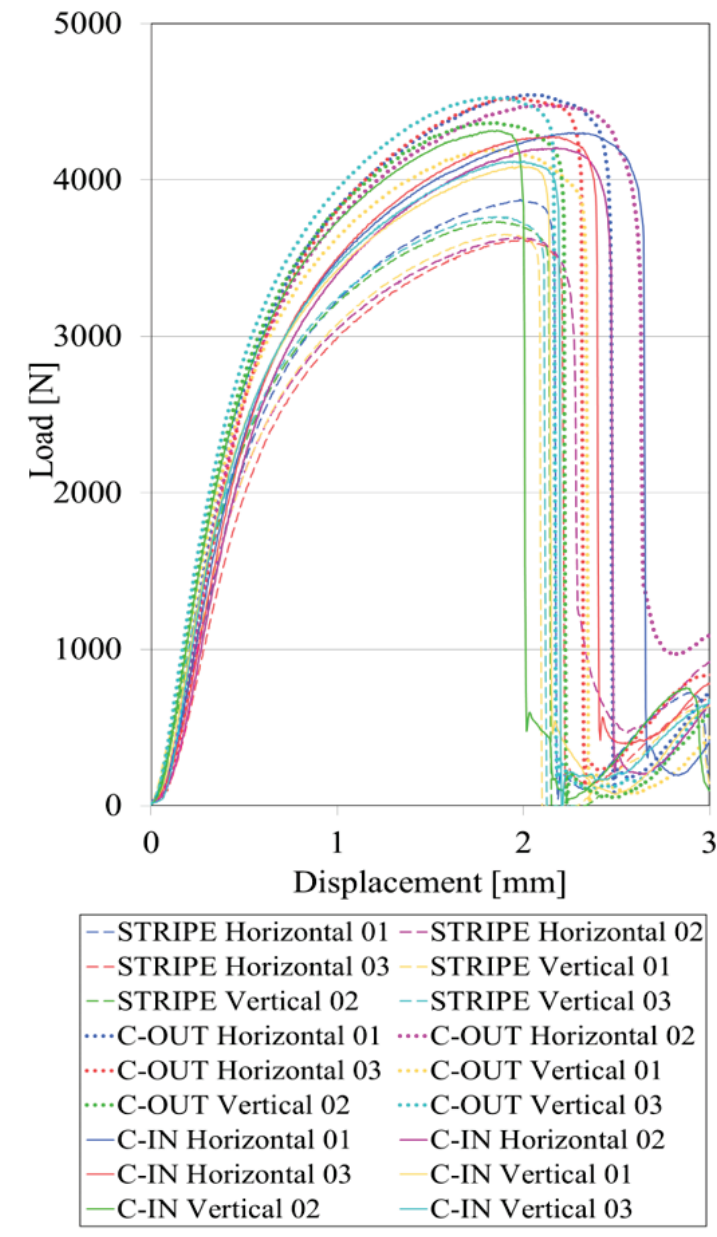

(b)

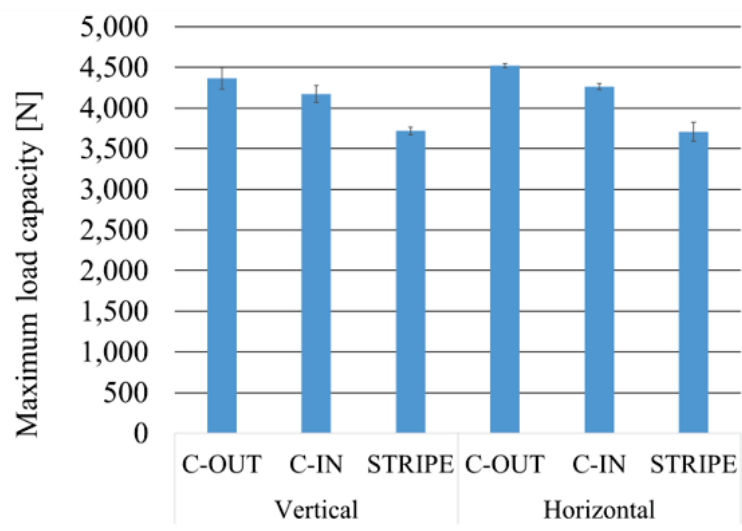

Fig. 4 Compressive properties of: (a) Load-displacement curve, (b) maximum load capacity of each compressive direction 


\subsection{Evaluation of the influence of scan order on concentric scan pattern}

Fig. 5 shows optical micrographs with vertical crosssections of the strut from C-OUT and C-IN. The boundaries of a melt pool can be sharply defined at the side and upper zone of the strut of C-OUT, as well as at the center and upper zone of the strut of C-IN. This indicates that different microstructures were developed depending on the scan order. (a)

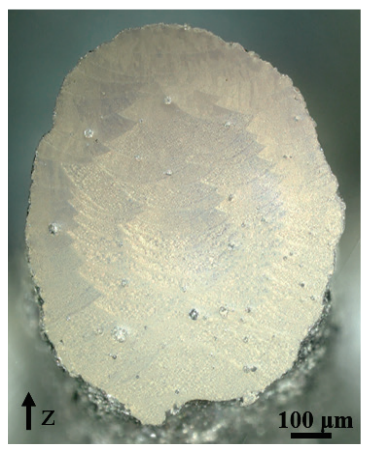

(b)

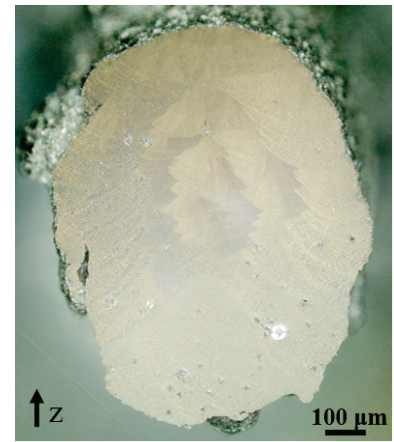

Fig. 5 Optical micrographs of the strut in a vertical cross-sectional view: (a) C-OUT, (b) C-IN
Fig. 6 shows EBSD grain boundary maps of C-OUT and C-IN. Notably, a number of holes in the lower zone of the strut were formed during the polishing process of OP-S with colloidal silica.

Both results reveal that the grain size in the upper zone of both struts was finer than that in the lower zone. By comparing the EBSD grain boundary maps, it was found that the grain size at the center of the strut of C-OUT was finer than that at the center of C-IN. (a)

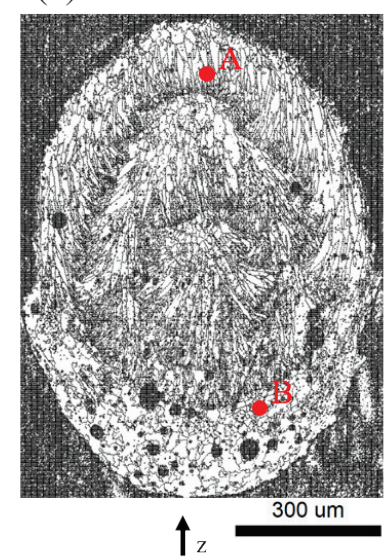

(b)

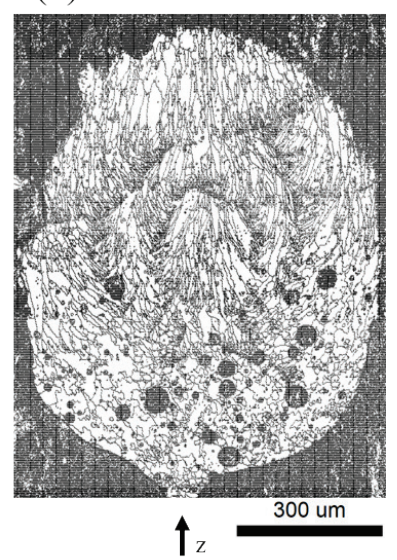

Fig. 6 EBSD grain boundary maps of the strut in a vertical cross-sectional view: (a) C-OUT, (b) C-IN. In the maps, grain boundaries are shown at a lower critical misorientation angle of $15^{\circ}$. Red points indicate regions observed with higher magnification in Fig. 7.

To examine the difference in the microstructure between the fine- and coarse-grained regions, we observed typical areas of the fine- and coarse-grained regions using SEM with a higher magnification. Fig. 7 shows SEM images with the microstructures of C-OUT observed at the fine-grained region (point A) and the coarse-grained region (point B) of Fig. 6. As shown in Fig. 7(a), finer Si particles are dispersed between the fine columnar $\alpha$-Al grains. On the other hand, in Fig. 7(b) coarser Si particles are formed in the dendrite arm spacing of the coarse $\alpha-\mathrm{Al}$ grains.

Table 1 shows the Vickers hardness test results from the upper and lower zones of the strut. The difference in the hardness of C-OUT and C-IN originates from the grain size: with the hardness of the fine-grained region being higher than that of the coarse-grained region. This result is consistent with a previous study on other aluminum alloys [7]. (a)

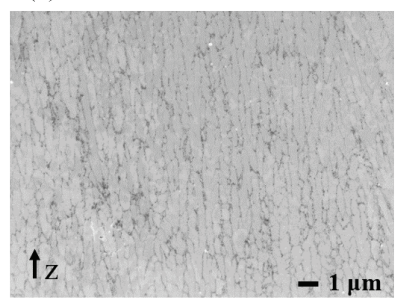

(b)

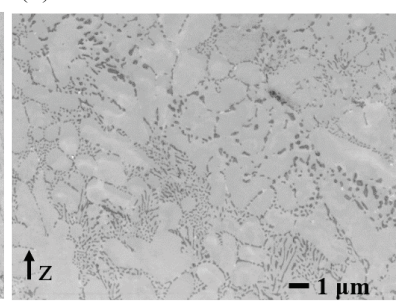

Fig. 7 Microstructures of the strut in a vertical cross-sectional view: (a) upper strut zone at point A of Fig. 6, (b) lower strut zone at point $\mathrm{B}$ of Fig. 6

Table 1 Vickers hardness

\begin{tabular}{ccc}
\hline & Upper zone & Lower zone \\
\hline C-OUT & $117 \pm 3$ & $81 \pm 12$ \\
\hline C-IN & $115 \pm 3$ & $79 \pm 9$ \\
\hline STRIPE & $116 \pm 7$ & $86 \pm 6$ \\
\hline
\end{tabular}

Furthermore, the results shown in Fig. 6 indicate that the area fraction of the fine-grained region of C-OUT is higher than that of $\mathrm{C}$-IN. This suggests that the strength of the strut of C-OUT is higher than that of C-IN, representing the difference between the load capacities of two lattice structures.

The area fraction of the fine-grained region was affected by the scan order of the concentric scan strategy. Fig. 8 illustrates the direction of heat transfer of the concentric scan strategies based on different scan orders. At each layer, the scan pattern was presented by three scan paths. In the first scan, the heat transfer direction was solely downwards. In the second and third scans, the (a)

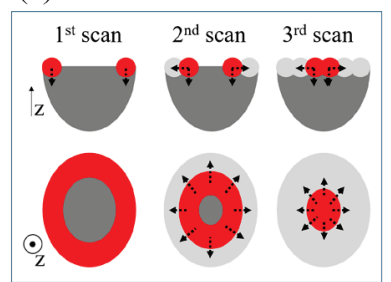

(b)

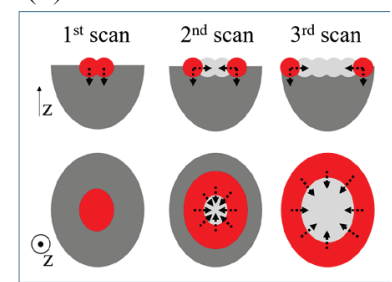

Fig. 8 Schematic illustrations of concentric strategy heat transfer in: (a) C-OUT, (b) C-IN. Powder in red is melted and solidified by each scan path, leaving heat transfer metallic object in light gray. Metallic object in dark gray underneath the current scan layer also provides heat transfer path, as illustrated by the arrows. 
direction of heat transfer was oriented downwards and widthways. Therefore, different scan orders resulted in a varying volume fraction and position of the metallic object acting as a heat sink. In the case of C-OUT, for instance, the temperature at the center of the strut increased by the third scan. Then, it quickly decreased due to heat transfer in both downward and lateral directions, resulting in the finer grain size. Heat transfer in the lateral direction was via the structure that was fabricated by the first and second scans with a much larger volume fraction than that of the third scan. In contrast, in the case of C-IN, the temperature at the center of the strut increased from the start of the first scan, and then, increased by the second and third scans, resulting in coarsening of the grain size. This suggests that the strength of the strut of C-OUT was higher than that of CIN, which resulted in the difference between the load capacities of two lattice structures.

\subsection{Evaluation of the influence of scan pattern}

Fig. 9 shows the cross-sectional shapes of the STRIPE strut. It is visible that STRIPE possesses irregular geometry, which is different from the ellipsoidal crosssection. Due to the nonuniform cross-section with large surface roughness, the area of the strut affected by the load capacity was reduced.

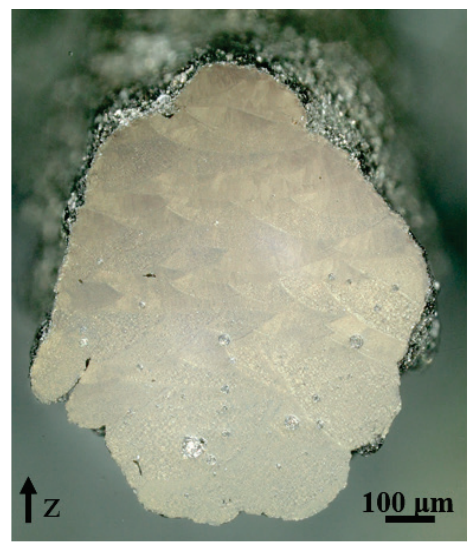

Fig. 9 Strut of STRIPE in a vertical cross-sectional view

More detailed investigation of the microstructures and hardness of the struts was conducted for C-OUT and STRIPE. Fig. 10 shows an EBSD grain boundary map of a vertical cross-section of the STRIPE strut. Most grains were classified as fine-grained in this cross-section. Therefore, the coarse-grained region was not sharply defined in the STRIPE sample, unlike in the C-OUT sample, as shown in Fig. 6(a). Grain size in the lower zone of the strut was found to be smaller than that of C-OUT in Fig. 6(a).

From Table 1, Vickers hardness in the lower zone of the strut of STRIPE was found to be higher than that of COUT. The increased hardness of STRIPE suggests that the strength of the strut of STRIPE was higher than that of COUT.

To investigate the microstructure differences between C-OUT and STRIPE, the grain orientations were evaluated. Fig. 11 shows EBSD inverse pole figure (IPF) map and a pole figure $(\mathrm{PF})$ vertical cross-section of C-OUT. In Fig. 11 (a), the colors represent grain orientation. The red color

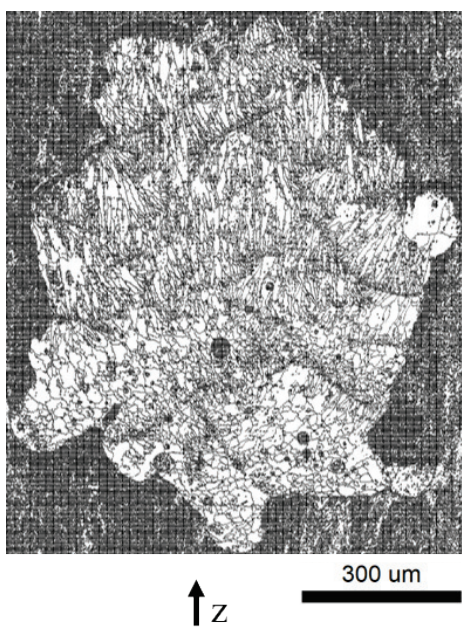

Fig. 10 EBSD grain boundary map of the strut of STRIPE in a vertical cross-sectional view. In the maps, grain boundaries are shown at a lower critical misorientation angle of $15^{\circ}$. (a)

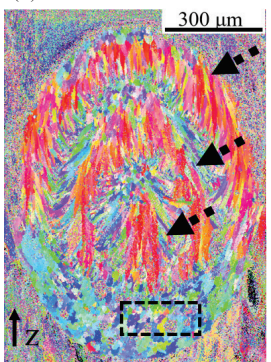

(b)

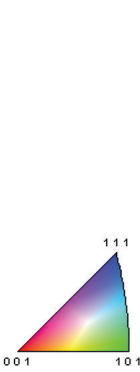

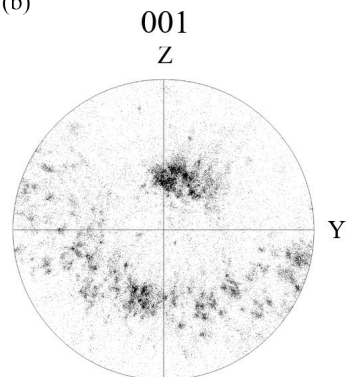

Fig. 11 (a) EBSD IPF map, and (b) PF of the strut of C-OUT in a vertical cross-sectional view. In (a), the colors represent grain orientation. Red color indicates that the orientation of the grain was 001 and parallel to the Z-direction. Black dotted arrows indicate three main red zones that are separated by the areas with distinct orientation. Black dotted square represents an area, where PF was obtained (b)

indicates that the orientation of the grain was 001 , i.e., parallel to the Z-direction. In Fig. 11(a), in the upper zone of the strut, the red grains were mainly distributed in three zones indicated by black dotted arrows. The preferential orientation of the red regions was parallel to the $\mathrm{Z}$ direction. However, at the boundaries of these red zones, no preferential orientation was found to be parallel to the Z-direction. The locations of the red zones were spatially overlapped with the locations of the laser scan paths. The difference in the observed grain orientations was explained by the curved boundaries of a melt pool, as mentioned in a previous study [8].

Fig. 11(b) shows the distribution of grain orientations. In the lower zone of the strut in Fig. 11(b), the preferential orientations were found to be around $35^{\circ}$ to the horizontal plane, consistent with the angle of the strut of $35.3^{\circ}$. This indicates that the heat transfer direction was along the strut at its lower zone. It also implies that the grains were columnar and anisotropic, displaying different mechanical properties.

Fig. 12 shows an EBSD IPF map and a PF of a vertical cross-section of STRIPE. In the upper zone, the location of the grains exhibiting orientation parallel to the Z-direction 
varies because the scan path was rotated by $67^{\circ}$ after passing each layer. In the lower zone, on the other hand, no grains possess any preferential orientation, suggesting that the grains were equiaxed. Therefore, STRIPE is preferable from the microstructural point of view as equiaxed grains are suitable for the isotropic mechanical properties.
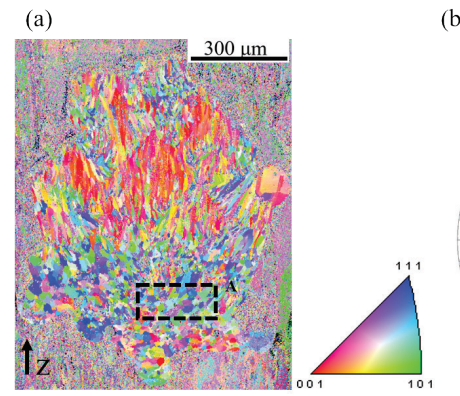

(b)

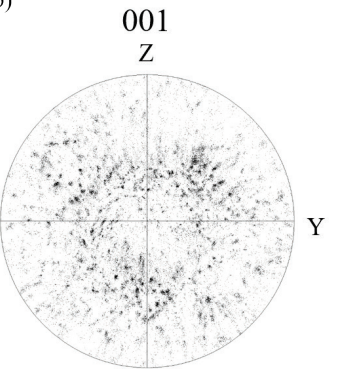

Fig. 12 (a) EBSD IPF map, and (b) PF of the strut of STRIPE in a vertical cross-sectional view. In (a), the colors represent grain orientation. Red color indicates that the orientation of the grain was 001 and parallel to the Z-direction. Black dotted square represents an area, where PF was obtained (b)

From the above-mentioned results, C-OUT provides the preferable heat direction from the inside to the outside. It prevents grain coarsening at the center of the strut and leads to higher geometrical accuracy. In addition, STRIPE provides smaller and equiaxed grains. Therefore, it is recommended to use a combination of C-OUT for the outside and STRIPE for the inside to improve the load capacity.

\section{Conclusions}

The investigation of the mechanical properties of metallic lattices revealed that C-OUT had a higher load capacity than C-IN due to the higher area fraction of the finegrained region. The coarsening of grains at the center of the strut occurred in C-IN. The mechanism was explained by the direction of heat transfer during fabrication. The scan pattern also affected the hardness of the lower zone of the strut and its geometrical accuracy. Although the hardness of STRIPE was higher than that of C-OUT due to the finer grain size, the load capacity of STRIPE was lesser than that of C-OUT, which was caused by the low geometrical accuracy of STRIPE. Therefore, the presented results suggest that load capacity can be improved by using a combined scan strategy of C-OUT and STRIPE.

\section{References}

[1] R. Mahshid, H. Hansen, and K. Højbjerre: Mater. Des, 104, (2016), 276

[2] N. Read, W. Wang, K. Essa and M. Attallah: Mater. Des, 65, (2015), 417

[3] Z. Hong, P. Tao, and X Shuangmei: Procedia CIRP, 61, (2017), 606

[4] R. Casati, J. Lemke, and M. Vedani: J. Mater. Sci., 32, (2016), 738

[5] S. Catchpole-Smith, N. Aboulkhair, L. Parry, C. Tuck, I.A. Ashcroft, and A. Clare: Addit. Manuf., 15, (2017), 113
[6] E. Olakanmi, R. Cochrane, and K. Dalgarno: Prog. Mater. Sci, 74, (2015), 401

[7] P. Delroisse, P.J. Jacques, E. Maire, O. Rigo, and A. Simar: Scr. Mater., 141, (2017), 32

[8] N. Takata, H. Kodaira, K. Sekizawa, A. Suzuki, and M. Kobashi: J. Jpn. Inst. Light Met., 67, (2017), 582

(Received: May 17, 2019, Accepted: January 20, 2020) 